\title{
PER UNA DEFINIZIONE INTERDISCIPLINARE DI 'NORMA'
}

\author{
di Edoardo Fittipaldi ${ }^{*}$
}

\author{
Mihi a docto doctore \\ Demandatur causam et rationem quare \\ Opium facit dormire. \\ A quoi respondeo, \\ Quia est in eo \\ Vertus dormitiva, \\ Cujus est natura \\ Sensus assoupire \\ (Bachelerius)
}

\section{Tre definizioni sociologico-giuridiche di norme e i loro problemi}

In questo articolo lo scopo che mi propongo è superare alcuni limiti di alcune importanti definizioni di 'norma' che sono state proposte in ambito sociologico-giuridico, sostituendole con una definizione che risulti capace di andare incontro alle esigenze, non solo della sociologia del diritto, ma anche dell'antropologia e dell'etologia. Farò qui largo uso di nozioni psicologiche, ma il mio scopo, come ho detto, non è dare un contributo alla psicologia, bensì di proporre un concetto che possa fungere da strumento per ricerche interdisciplinari.

La definizione - ovviamente stipulativa - di 'norma' che proporrò è la seguente: con 'norma' deve intendersi la disposizione stabile a provare emozioni etiche nel caso in cui si diano determinate circostanze.

Ma prima di esaminare la definizione qui proposta mi pare necessario esaminare brevemente tre importanti definizioni di 'norma' proposte da tre sociologi del diritto: Theodor Geiger, Niklas Luhmann e Vincenzo Ferrari.

Fra le - invero non moltissime - definizioni esplicite di 'norma' proposte dai sociologi del diritto ho scelto queste poiché sono semplici ${ }^{1}$ e perché non fanno ricorso in alcun modo alla linguisticità ${ }^{2}$.

Le concezioni linguistiche della norma sono, non solo incompatibili con qualsivoglia tentativo di gettare un ponte fra la sociologia del diritto e

\footnotetext{
* Dipartimento di Studi giuridici ecclesiasticistici, filosofico-sociologici e penalistici "Cesare Beccaria”, Università degli Studi di Milano.

${ }^{1}$ Rehbinder (2000, § 37), per esempio, ha fornita una definizione piuttosto articolata di norma. Tuttavia, sia a causa della sua non semplicità sia a causa del debito di essa nei confronti delle Vorstudien di Geiger, questa definizione non verrà discussa in questa sede.

${ }^{2}$ Fa purtroppo ricorso a una definizione linguistica Lawrence Friedman (1975: 26).
} 
l'etologia, ma altresì incapaci di rendere conto di fenomeni come i crittotipi (Sacco 1991: 384 ss. e 1992), il diritto muto (Sacco 1995), le norme latenti (Geiger 1964²: 96 ss.), tutti fenomeni di straordinaria importanza sociologico-giuridica.

Theodor Geiger (1964²: 69 ss.) definì la norma (Norm) nel modo seguente (uso un formalismo più semplice del suo): $c \rightarrow[b \underline{\vee}(\neg b \rightarrow r)]$

In altri termini, a suo avviso, una norma consiste nel fatto che, qualora abbia luogo la fattispecie concreta $c$, o ha luogo il comportamento $b$ oppure, nel caso in cui il comportamento $b$ non abbia luogo, ha luogo la reazione $r$. Per Geiger, l'esistenza ${ }^{3}$ di una norma è questione di grado. Egli definì questo grado nel modo seguente: $g={ }^{e} / c$. La lettera $g$ esprime, appunto, il grado di esistenza della norma. La lettera $e$ esprime il numero di casi in cui la norma è efficace, e tale numero è la somma dei casi in cui, essendo stata integrata la fattispecie $c$, o si è potuto osservare il comportamento $b$ o, qualora il comportamento $b$ non sia stato tenuto, si è potuta osservare la reazione $r$. In entrambi i casi, come ho detto, è necessario che sia stata integrata la fattispecie $c$.

In questo modo Geiger credette di essere stato in grado di sbarazzarsi della psicologia. Ciò che egli fa, invece, è mescolare l'ansia sociale con l'ansia etica (o normativa) impedendo al sociologo di distinguere fra questi fenomeni. ${ }^{4}$

Geiger sembra aver pensato che la causa, etica o non etica, per cui un individuo si comporta in un certo modo fosse irrilevante per la sociologia del diritto. Per lui è irrilevante se questa causa sia la paura di una reazione da parte altrui, o il fatto di anticipare una personale esperienza di vergogna o di senso di colpa in caso di una certa condotta, ecc.

Un altro problema della definizione di Geiger è che sembra essere stata concepita esclusivamente per norme ipotetiche, e quindi non è chiaro come possa venire utilizzata nel caso di norme categoriche come la proibizione dell'omicidio. Come dobbiamo calcolare il numero di casi in cui, potendo compiere un omicidio, ci asteniamo dal farlo? Dobbiamo calcolare tutti i casi in cui stiamo semplicemente guidando e potremmo uccidere un pedone semplicemente sterzando all'improvviso?

Queste sono solo alcune delle ragioni per cui, a mio avviso, una definizione come quella di Geiger è inutilizzabile da parte della sociologia del diritto. Ulteriori ragioni emergeranno nel corso di questo lavoro. Si tratta di ragioni legate alla sterilità euristica della definizione proposta da Geiger.

\footnotetext{
${ }^{3}$ Geiger usa i termini Wirklichkeit ("realtà") e Verbindlichkeit ("obbligatorietà"), e sembra usare tali termini come sinonimi.

${ }^{4}$ Per una discussione più ampia di questo argomento mi permetto di rinviare al mio Fittipaldi 2012a (102 s.).
} 
Veniamo ora alla definizione proposta da Niklas Luhmann. Com'è noto Luhmann definì la norma come aspettativa stabilizzata controfattualmente. La delusione dell'aspettativa è irrilevante rispetto al nutrire l'aspettativa $\left(1969^{*}\right)$.

Questa definizione, rispetto a quella di Geiger, sembra tenere presente la psicologia. Il concetto cruciale è qui quello di aspettativa (Erwartung). Come si vede, questa aspettativa, stando alla definizione di Luhmann, potrebbe sopravvivere anche nel caso in cui il grado di esistenza di cui parla Geiger $\left({ }^{e} / c\right)$ fosse pari a 0.

Un primo difetto di questa definizione luhmanniana è che essa sembra presupporre almeno due individui: colui che nutre l'aspettativa $\mathrm{e}$ l'individuo la cui condotta è oggetto dell'aspettativa. Ne segue che questa definizione sembra escludere la possibilità per il sociologo del diritto di investigare $i$ casi in cui a essere oggetto di aspettativa sia la stessa condotta o gli stessi pensieri del soggetto che l'aspettativa la nutre. Per quanto siffatti fenomeni possano a primo acchito sembrare di interesse esclusivo dello psicologo, è del tutto evidente che tali fenomeni psichici diventano immediatamente sociologici non appena ci si interroghi sulla variabilità di essi in diverse culture oggetto di indagine. Del resto, a proposito del suicidio, a suo tempo Emile Durkheim ebbe modo di osservare che già i diversi tassi di questo fenomeno nelle diverse culture fanno di esso un fenomeno meritevole di essere investigato sociologicamente; e ciò a dispetto della sua natura prima facie eminentemente privata $\left(1897^{*}: 77\right)$.

Un secondo difetto della definizione di Luhmann è che non è chiaro che tipo di fenomeno psichico siano le aspettative. Dare una risposta a questa domanda è uno degli obiettivi di questo articolo.

Passiamo infine alla definizione di Vincenzo Ferrari, definizione che sarà il mio punto di partenza:

La norma [...] è un modello al quale un'azione si rapporta, oppure può rapportarsi, oppure deve rapportarsi. Una norma può esaurire la sua funzione nella sfera psichica del soggetto agente senza venire a conoscenza di altri. Molte nostre azioni

s'ispirano a modelli che non comunichiamo, spesso neppure a noi stessi. Tali modelli, centrali nell'analisi psicologica, possono interessare indirettamente anche il sociologo, sia perché, inducendo all'azione, producono effetti sociali, sia perché alle loro radici vi sono spesso norme socialmente diffuse che il soggetto ha interiorizzato. [Ferrari 2005: 42, tutti i corsivi sono miei eccetto il primo]

Secondo Ferrari le norme puramente psichiche sono rilevanti per il sociologo, ma solo indirettamente. A suo avviso, affinché le norme divengano di diretto interesse per il sociologo del diritto esse debbono essere comunicate (ibidem). 
A mio avviso due sono le ragioni che militano contro una siffatta restrizione.

Anzitutto, i fenomeni di diritto muto investigati da Rodolfo Sacco (cfr. supra), a mio avviso, sono di diretto interesse per il sociologo del diritto. Inoltre, una definizione di norma che esiga anche la comunicazione impedisce di gettare ponti fra la sociologia del diritto e l'etologia. Infine, come abbiamo già detto richiamando Durkheim, non occorre un'interazione fra individui per qualificare un fenomeno come un fenomeno di interesse sociologico.

Per queste ragioni il mio punto di partenza sarà la definizione psicologica di norma proposta da Ferrari, e non la sua definizione sociologica.

Ora, la definizione di Ferrari pone alcuni problemi che in questo scritto cercherò di affrontare:

1. Che tipo di entità è il modello di cui parla Ferrari?

2. Quali sono i modi in cui il soggetto si rapporta col modello? In altri termini, quali sono i rapporti fra questi modi e le cosiddette "modalità deontiche"?

3. Che cosa vuol dire il verbo indurre usato da Ferrari?

4. Che cos'è l'interiorizzazione di cui parla Ferrari?

Per quanto riguarda la prima questione, la mia proposta è di intendere il modello, non come un'entità ideale (entità cui chi scrive non crede neanche nel caso delle matematiche), bensì come un fenomeno psicologico; in altri termini, intendo il modello come la rappresentazione di una certa condotta. La condotta oggetto di rappresentazione può essere una condotta dello stesso autore della rappresentazione ma anche la condotta di altri soggetti. In quest'ultimo caso l'autore della rappresentazione potrà essere un terzo oppure un avente diritto (nel caso in cui non si tratti di un Recht auf eigenes Verhalten - cfr. infra)

Vedremo che in molti casi questo modello emerge a contrario come sottoprodotto del funzionamento delle emozioni etiche (o normative).

$\mathrm{Ne}$ segue che è dalle emozioni etiche che dobbiamo partire.

In questo modo cercherò di rispondere alle questioni (2), (3) e (4), e al tempo stesso proporrò una riduzione - o meglio, una scomposizione - psicologica del concetto di norma ${ }^{5}$.

Il mio punto di partenza è l'ipotesi che le emozioni etiche non sono innate: le emozioni etiche emergono nel corso dell'interazione del bambino con le sue figure di accudimento (per lo più i suoi genitori). Non tutti i bambini le sviluppano. I Wolfskinder sembrano non svilupparle, ad esempio. Fermo

\footnotetext{
${ }^{5}$ Per quanto riguarda la questione (3) non esaminerò i casi in cui le norme incidono causalmente sul comportamento in modi diversi dall'induzione alla conformità (operanza o praxeotropismo). Su questi temi si veda Conte 2011.
} 
rimane che non intendo affatto escludere la possibilità che certi animali non-umani sviluppino alcune emozioni etiche (cfr. infra).

Prima di esaminare le emozioni etiche va sottolineato che nella sua definizione Ferrari omette - credo intenzionalmente - la distinzione fra comportamento etico e comportamento economico. A mio avviso, come ho già anticipato parlando di Geiger, il sociologo del diritto ha a che fare con fenomeni completamente diversi:

1. nel caso in cui un individuo si astenga dal rubare perché considera sbagliato rubare, $\mathrm{e}$

2. nel caso in cui un individuo si astenga dal rubare poiché teme spiacevoli conseguenze.

Se vogliamo spiegare come mai certi individui adottino certi modelli di azione per loro economicamente svantaggiosi dobbiamo - almeno in certi casi - ipotizzare che le loro azioni siano causate da emozioni etiche. Vedremo inoltre che in certi casi non è l'azione dell'individuo a essere etica, bensì la reazione degli altri individui in casi di trasgressione (gli etologi parlano in questi casi di moralistic aggression).

Discuterò le seguenti emozioni: ira, indignazione, senso di colpa, vergogna, orgoglio e ammirazione. Fermo rimane che non si tratta di un elenco esaustivo.

Per riferirmi all'ira e all'indignazione, nel prosieguo, parlerò di emozioni etiche di tipo sadico. Ciò perché esse consistono in uno scarico di aggressività rivolto verso individui diversi dal soggetto. Userò l'espressione emozioni etiche di tipo masochistico per riferirmi alla vergogna e al senso di colpa. La scelta di questa espressione è un ovvio tributo a Freud, dal momento che il grande studioso ipotizzò che il senso di colpa traesse la propria "energia" dall'aggressività del soggetto; un'aggressività rivolta verso il soggetto stesso. Non è necessario aderire a quest'ipotesi (chi scrive non vi aderisce) per adottare questa terminologia, esattamente come non occorre aderire a una certa ipotesi eziologica per adottare il termine isteria. Infine, userò l'espressione emozioni etiche di tipo narcisistico per riferirmi all'orgoglio e all'ammirazione (anche qui il riferimento freudiano è ovvio).

Nel prossimo paragrafo mi occuperò delle emozioni etiche di tipo sadico. Nel $\S 3$ mi occuperò di quelle di tipo masochistico. Nel $\S 4$, infine, mi occuperò di quelle narcisistiche. Come si vedrà, il $\S 2$ è di dimensioni molto maggiori rispetto ai $\S \S 3$ e 4 . Ciò è due dovuto al fatto che, da un lato, la letteratura su questi fenomeni è meno coesa di quella sugli altri fenomeni, dall'altro al fatto che il ruolo delle emozioni etiche sadiche è di interesse assolutamente centrale per il sociologo del diritto, fermo rimanendo che l'importanza delle emozioni etiche di tipo masochistico e di tipo narcisistico non può essere sottovalutata. 


\section{Disposizioni etiche di tipo sadico}

Uno dei principali obiettivi delle figure di accudimento in tutte le culture umane sembra essere questo: insegnare al bambino a contenere la propria aggressività altrimenti illimitata. (Con questa affermazione, beninteso, non voglio escludere che vi siano specie di animali non umani nelle quali gli adulti insegnano ai piccoli il contenimento dell'aggressività).

Per quanto ne sappia, il primo studioso ad aver fatto questa osservazione fu Adam Smith:

A very young child has no self-command; but, whatever are its emotions, whether fear, or grief, or anger, it endeavours always, by the violence of its outcries, to alarm, as much as it can, the attention of its nurse, or of its parents. While it remains under the custody of such partial protectors, its anger is the first and, perhaps, the only passion which it is taught to moderate. [Smith $1790^{6}$ : Parte 3, Cap. 3, § 22]

Smith era convinto che la gran parte delle emozioni etiche ("moral sentiments") si sviluppasse durante la socializzazione secondaria. Secondo lui, al più, la socializzazione primaria aveva la funzione di insegnare il contenimento dell'aggressività. Nonostante la sottovalutazione smithiana della socializzazione primaria sia oggi insostenibile, rimane un'ipotesi altamente plausibile che una delle prime cose che le figure di accudimento insegnano al piccolo sia il contenimento dell'aggressività.

Il passaggio dall'aggressività illimitata all'aggressività contenuta costituisce al tempo stesso l'instaurazione delle prime emozioni etiche, cioè le emozioni etiche di tipo sadico. Con questa instaurazione, infatti, il piccolo apprende che può scaricare la propria aggressività solo in determinate circostanze, e non in qualunque momento.

Queste circostanze sembrano essere le seguenti:

1.1. un attacco al corpo;

1.2. un impedimento alla libertà di movimento;

2. il fatto che scaricare l'aggressività non comporti reazioni da parte dei genitori.

A mio avviso le circostanze (1.1) e (1.2) costituiscono i paleotipi ${ }^{6}$ di quelle che chiamo ira domini e ira iuris. Per quanto riguarda la (2) mi pare essere il paleotipo dell'indignazione.

\footnotetext{
${ }^{6}$ Uso il termine paleotìpo anziché protòtipo poiché i paleotipi si riferiscono a situazioni infantili che presso gli adulti possono anche essere molto diverse dalla situazioni che un adulto additerebbe come "best examples" (uso il linguaggio della teoria dei prototipi). A mio avviso il concetto di paleotipo (che presuppone un approccio di tipo psicoanalitico) è compatibile con un approccio di tipo psicologico-cognitivo che faccia uso dei prototipi qualora si sostenga che i prototipi sono utili per formulare previsioni e spiegazioni senza sostenere che l'unica origine possibile per i prototipi sia la ripetizione di passate esperienze (come so-
} 


\section{Iniziamo con l'ira.}

Come ho detto, vi sono due tipi di ira: l'ira domini e l'ira iuris. I termini sono miei, la distinzione non lo $\mathfrak{e}^{7}$ :

The cognitive antecedents that initiate anger [...] can be summarized as follows: Something (usually another person [...]) interferes with the person's execution of plans or attainment of goals (by reducing the person's power, violating expectations, frustrating goal-directed activities). Alternatively, the person perceives another as harming him or her in some way (inflicting physical or psychological pain). [Shaver et al. 1987: 1077, corsivi miei]

Il fatto che i due tipi di ira possano sovrapporsi, in quanto collegate al corpo, non esclude che si possa tenerle concettualmente distinte.

Un'importante differenza funzionale fra i due tipi di ira consiste nel fatto che nel caso di ira domini il soggetto (Ego) mira a fare del male a un altro individuo (Alter), mentre nel caso di ira iuris Ego si focalizza prevalentemente sulla propria azione; un'azione che Ego vuole realizzare nonostante il tentativo di Alter di impedirla.

Il paleotipo dell'ira iuris spiega, a mio avviso, come mai sembri che il latino ius potesse essere utilizzato esclusivamente per riferirsi a un'attività dell'avente diritto, e non dell'obbligato ${ }^{8}$. Questa è la ragione per cui ho scelto il termine ius per riferirmi a questo tipo di ira. Questo paleotipo può forse spiegare come mai in numerose lingue l'idea di diritto sia collegata con l'idea di dritto. Per quanto riguarda invece l'uso di dominus per qualificare il secondo tipo di ira la ragione è che mi sembra che il paleotipo della proprietà possa essere ravvisato nella proprietà delle proprie membra 9 .

Esaminiamo ora l'ira domini.

La norma per cui Alter non può far del male a Ego può venire ridotta alla stabile disposizione di Ego a scaricare la propria (usualmente contenuta) aggressività nel caso in cui Alter si accinga a fargli del male. Adotterò il seguente formalismo:

$$
\text { (1) (nuocere-a-Ego })_{\text {alter }} \rightarrow \mathrm{IRA}_{\text {ego }}
$$

stenuto, ad esempio da Shaver et al. 1987: 1072). Mi sembra che i paleotipi possano svolgere un ruolo assai importante ai fini della spiegazione del modo in cui i prototipi emergono negli adulti. In altre parole la capacità predittiva ed esplicativa dei prototipi non esclude che tali capacità possano essere a loro volta spiegate per mezzo di teorie diverse da teorie psicologico-cognitive. Per quanto riguarda il termine archetipo, invece, esso non verrà utilizzato qui giacché mi focalizzerò prevalentemente su questioni ontogenetiche.

${ }^{7}$ La distinzione fra ira domini e ira iuris mi pare confrontabile anche la distinzione fra aggressione reattiva e aggressione strumentale (cfr. Ceretti \& Natali 2009: 70).

${ }^{8}$ Per una discussione della letteratura romanistica in materia si veda Fittipaldi 2012a (248 s.).

${ }^{9}$ Cfr. Fittipaldi 2012a (\$ 4.12). 
In questo modo il più generale fenomeno della norma giuridica ${ }^{10}$ proibitiva può essere ridotto alla seguente disposizione ${ }^{11}$ :

(2) $x_{\text {alter }} \rightarrow \mathbb{R A}_{\text {ego }}\left(x_{\text {alter }}\right)$

Il fenomeno reale, qui, non è la supposta norma giuridica proibitiva bensì il potenziale scarico di aggressività da parte di Ego qualora Alter compia un certo tipo di azione.

Gli obblighi giuridici possono essere formalizzati nel seguente modo:

(3) $\neg x_{\text {alter }} \rightarrow \mathbb{R A}_{\text {ego }}\left(\neg x_{\text {alter }}\right)$

In questo caso il fenomeno reale non è l'obbligatorietà della condotta $x$ bensì il fatto che Ego scarichi la propria aggressività in caso di non- $x$ (formalizzato come $\neg x$ ). Ne segue che per l'esistenza di un siffatto fenomeno giuridico è più che sufficiente la disposizione di Ego a provare ira in determinate circostanze.

In questi casi il modello di cui Ferrari parla nella sua definizione di norma (supra § 1) non è che un sottoprodotto del fatto che Ego abbia la disposizione a scaricare la propria aggressività usualmente repressa nel caso in cui Alter tenga o non tenga una certa condotta. Il modello emerge a contrario; e ciò accade nel caso in cui Ego sia in grado di razionalizzare le situazioni che in lui fanno sì che scarichi la propria aggressività.

$\mathrm{Ne}$ segue che anzitutto vi sono situazioni in cui gli individui scaricano la propria aggressività usualmente repressa (quella che qui chiamo ira) e solo in seguito è possibile che emerga un modello di azione (negativo rispetto a quelle situazioni) che il soggetto si rappresenta come obbligatorio o vietato.

Veniamo ora all'ira iuris.

Parlo di ira iuris nel caso in cui il paleotipo sia costituito dalla focalizzazione del soggetto, non su un'azione o un'inazione altrui, bensì su un'azione o un'inazione propria.

Abbiamo due situazioni tipiche che possono dare luogo all'ira iuris:

1. il caso in cui Alter tenti di interferire con l'attività di Ego;

2. il caso in cui Alter mostri sintomi di intolleranza dell'attività di Ego, senza che ciò comporti da parte di Alter un tentativo di interferire con l'attività di Ego.

Mi riferirò a entrambi questi fenomeni in Alter col termine nonpati (mutuato da Petrażycki 1909-10; che scrivo tuttavia nonpati anziché non pati per esigenze di formalizzazione). Per quanto riguarda il caso (2) si pensi a

${ }^{10}$ Per quanto riguarda la distinzione tra fenomeni giuridici e fenomeni morali, adotto la distinzione stipulativa proposta da Petrażycki (1909-10 \& 1909-10*). Cfr. anche Fittipaldi 2012a (cap. 4) e 2012b.

${ }^{11}$ Non specificherò più stabile, nel prosieguo, giacché si tratta di un pleonasmo. 
Tizio che si adiri alla sola idea che il vicino Caio possa non riconoscere la sua servitù di passaggio, fermo rimanendo che Caio, di fatto, nulla faccia per ostacolare Tizio nell'esercizio di essa. Esempi analoghi possono essere formulati pensando a libertà fondamentali quali la libertà di manifestazione del pensiero. Possiamo formulare questi fenomeni nel modo seguente:

(4) $\operatorname{NONPATI}_{\text {alter }}\left(y_{\text {ego }}\right) \rightarrow \operatorname{IRA}_{\text {ego }}\left(\operatorname{NONPATI}_{\text {alter }}\left(y_{\text {ego }}\right)\right)$

(5) $\operatorname{NONPATI}_{\text {alter }}\left(\neg y_{\text {ego }}\right) \rightarrow \operatorname{IRA}_{\text {ego }}\left(\operatorname{NONPATI}_{\text {alter }}\left(\neg y_{\text {ego }}\right)\right)$

Uso la lettera $y$ anziché la lettera $x$ per evidenziare il fatto che qui abbiamo a che fare con un Recht auf eigenes Verhalten; un fenomeno la cui esistenza è negata dalla gran parte dei teorici del diritto.

Per quanto riguarda il caso (5) mi si permetta di portare un esempio personale: la mia ira all'idea che qualcuno possa anche solo sollevare dei dubbi circa il mio diritto di suicidarmi digiunando.

È molto importante sottolineare che incompatibili esperienze di ira in Ego e Alter possono alimentarsi vicendevolemente:

(6) $\operatorname{IRA}_{\text {alter }}\left(y_{\text {ego }}\right) \rightarrow \operatorname{IRA}_{\text {ego }}\left(\operatorname{IRA}_{\text {alter }}\left(y_{\text {ego }}\right)\right)$

(7) $\operatorname{IRA}_{\text {alter }}\left(\neg y_{\text {ego }}\right) \rightarrow \operatorname{IRA}_{\text {ego }}\left(\operatorname{IRA}_{\text {alter }}\left(\neg y_{\text {ego }}\right)\right)$

La (6) si legge in questo modo: l'ira di Alter per la condotta $y$ di Ego causa l'ira di Ego per l'ira di Alter (relativa alla condotta di Ego stesso).

Ovvio che simili situazioni possono degenerare ${ }^{12}$.

Un tipico Recht auf eigenes Verhalten è lo ius puniendi.

Questo fenomeno può essere osservato quando chi punisce reagisce con ira a un sintomo del fatto che il soggetto punito non accetti intimamente la punizione.

Per quanto riguarda l'obbligo di pati, che tipicamente il soggetto punito deve riconoscere affinché il punitore non si adiri esso può consistere spesso quella forma di sollievo etico che è l'espiazione ${ }^{13}$.

La disposizione a provare ira svolge un ruolo fondamentale nel funzionamento della società. Questo punto è stato sintetizzato magistralmente da Schlicht, per cui mi limito a riprodurre le sue parole:

The effect of entitlements and obligations is most easily understood by looking at commonplace transactions. Consider, as an example, a taxi ride in an unfamiliar town. A person enters a taxi cab and tells the driver where to go. Upon arrival the taxi driver points to the taximeter and indicates the fare. The customer pays [...] .

The customer leaves, the taxi disappears. Neither party expects to meet the other

${ }^{12}$ È questo il cuore della natura polemogena dei fenomeni giuridici indicata da Petrażycki (1909-10 \& 1909-10*, cfr anche Fittipaldi 2012a e 2012b).

${ }^{13}$ Su questo argomento si veda Piaget 1932: 257, s., 1932*: 321 s., nonché Fittipaldi 2012a (181 ss.). 
again, and no-one thinks about the episode any further; this is the usual pattern of behavior. [...]

Why do both parties honor the contract? The passenger, for his part may consider leaving the taxi without paying, as he has been brought to the place where he wanted to go. He may fear, however, that this would infuriate the taxi driver. As the driver appears to be a strong and determined person, this appears too high a risk, and the passenger decides to pay.

However, after having handed over the money, nothing has changed in the mutual bargaining position: the taximeter reading is still the same, the physique of the taxi driver appears as threatening as before, and the passenger still has cash in his pocket. So why does the taxi driver not insist that the passenger pay again, and why shouldn't the passenger comply if he did before?

The taxi driver may think that such a demand would infuriate the passenger. The passenger could resist, and could consider legal action. So he is content with what he has obtained and drives away. [Schlicht 1998: 29 s.]

Una differenza importante fra la posizione qui sostenuta e quella sostenuta da Schlicht è che Schlicht - nello stesso libro da cui è tratta la citazione appena riportata - sembra escludere che fenomeni quali le pretese nevrotiche (neurotic claims) individuate da Karen Horney siano fenomeni di moralistic aggressions (Schlicht mutua questo termine dall'etologia).

La mia opinione è invece che le pretese nevrotiche siano fenomeni giuridici in piena regola, così come quelle che Horney chiama "secret claims toward life in general" (1950: 46).

Per chiarire il punto mi pare utile riprodurre un passo nel quale Horney descrive un episodio personale. Durante la seconda guerra mondiale - riporta la studiosa americana - le fu cancellato un volo da Corpus Christi a New York, e fu costretta a rientrare a New York in treno (si trattava di un viaggio di tre giorni).

Ecco come Horney descrive la propria esperienza:

Although I considered this regulation perfectly justified in principle, I noticed that I was furiously indignant $\left[{ }^{14}\right]$ when it applied to me. I was really exasperated at the prospect of a three-day train ride to New York, and became greatly fatigued. [Horney 1950: 44]

\section{Ed ecco la sua analisi:}

As long as I felt it to be an unfair imposition, it seemed almost more than I could endure. Then, after I had discovered the claim behind it - although the seats where just as hard, the time it took just as long - the very same situation became enjoyable. [...] Certainly there are experiences which are so severe to become crushing. But these are rare. For the neurotic, minor happenings turn into catastrophes and life becomes a series of upsets. [Horney 1950: 58]

\footnotetext{
${ }^{14}$ Nella terminologia da me adottata bisognerebbe usare il termine angry.
} 
Un fenomeno connesso con questo fenomeno è il diritto di essere un'eccezione (Ausnahmsanspruch) investigato da Sigmund Freud (1916). Non è questa la sede per discutere questo fenomeno.

Il cuore del concetto di ira come emozione etica è il concetto di aggressività usualmente repressa. Sorge la questione di come intendere quest'ultimo concetto.

La questione è analoga a quella discussa da Silverberg e Gray in ordine alla definizione di aggressione (che è ciò che qui chiamo scarico di aggressività). Questi due autori offrono la seguente definizione:

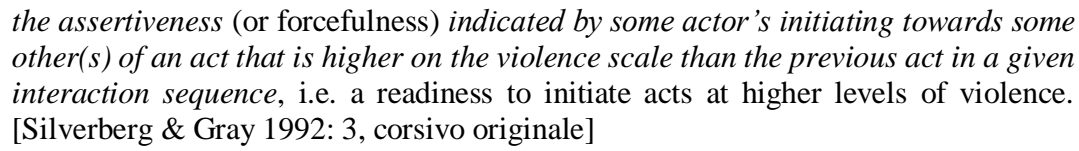

the assertiveness (or forcefulness) indicated by some actor's initiating towards some other(s) of an act that is higher on the violence scale than the previous act in a given interaction sequence, i.e. a readiness to initiate acts at higher levels of violence. [Silverberg \& Gray 1992: 3, corsivo originale]

Questa definizione prende in considerazione la sequenza delle interazioni. Si tratta di una soluzione più che compatibile con l'approccio qui proposto. Tuttavia mi pare opportuno segnalare la possibilità di situazioni nelle quali il livello di aggressività è, per così dire, strutturalmente (o costitutivamente) soprammedia. Situazioni del genere sono certe attività agonistiche.

Ora, mi parrebbe quanto mai inopportuno parlare di ira per descrivere il tipo di aggressività scaricata nelle attività agonistiche.

Per comprendere questo punto è utile esaminare brevemente queste situazioni.

Anzitutto occorre distinguere le attività sportive agonistiche in due tipi, contrapponendo gli sport-gara agli sport-partita. Definisco una competizione sportiva sport-partita come un'attività fisica svolta da almeno due squadre ${ }^{15}$, nella quale la vittoria dipende dal fatto di riuscire a svolgere determinate attività nell 'interazione con la o le squadre avversarie (che mira o mirano a svolgere le medesime attività). Per contro, definisco una competizione sportiva sport-gara come un'attività fisica da parte di almeno due squadre nella quale la vittoria dipende (quasi) esclusivamente dal fatto di riuscire a svolgere determinate attività (di regola, meglio degli altri partecipanti) $e$ a prescindere (o quasi completamente a prescindere) dall'interazione con lo o gli avversari. Nel caso degli sport-gara l'interazione con l'avversario è assente e il partecipante, di regola, si focalizza esclusivamente sulla propria prestazione ${ }^{16}$.

${ }^{15}$ Una squadra può essere composta anche da un solo individuo, nella definizione qui presentata.

${ }^{16}$ Invero, gli sport-gara possono essere spesso praticati anche da soli. In casi del genere l'unico partecipante può confrontare le proprie prestazioni attuali con le proprie prestazioni passate. 
Ora, mi pare ipotizzabile che specialmente nel caso di sport-partita i partecipanti possano sostenere la propria azione volta a superare l'interferenza avversaria con la propria attività per mezzo di un livello soprammedia di aggressività. Per distinguere questo tipo di scarico di aggressività mi pare utile utilizzare il termine impeto.

La differenza fondamentale fra l'impeto e l'ira mi pare essere che nel caso dell'impeto il soggetto scarica la propria aggressività per superare la resistenza avversaria ma nondimeno il soggetto non vive il nonpati avversario, in quanto tale, come un fattore di scarico della propria aggressività. ${ }^{17}$

Possiamo così parlare di un diritto soggettivo agonistico in contrapposto a un diritto soggettivo giuridico. Formalizzo i fenomeni psichici soggiacenti a tali diritti (che, beninteso, vanno intesi come ipostatizzazioni) nel seguente modo:

(8) $\operatorname{IMPETO}_{\text {ego }}\left(y_{\text {ego }}\right)$

(9) $\operatorname{NONPATI}_{\text {alter }}\left(y_{\text {ego }}\right) \rightarrow \operatorname{IRA}_{\text {ego }}\left(\operatorname{NONPATI}_{\text {alter }}\left(y_{\text {ego }}\right)\right)$

Nel caso dei diritti soggettivi agonistici abbiamo solo il fenomeno (8). Ovvio che può accadere che un giocatore - per esempio un calciatore - scarichi la propria aggressività in seguito al fatto che un giocatore avversario sia riuscito, pur senza commettere falli, a impedirgli di andare a rete. Stando alle proposte qui adottate una simile esperienza è un'esperienza di ira, e quindi va ricondotta a un'esperienza di diritto soggettivo di tipo giuridico, e non agonistico. Ma mi pare evidente che si tratta di fenomeni sporadici, che qualora divenissero sistematici meriterebbero la qualificazione di neurotic claim nel senso di Horney.

Esaminiamo ora l'indignazione.

Anche in questo caso abbiamo a che fare con uno scarico di aggressività usualmente repressa. Ma qui finisce la somiglianza con l'ira e l'impeto.

Se l'ira e l'impeto sono tipiche esperienze degli aventi diritto, l'indignazione è tipica dei terzi (ammesso che ve ne siano).

Se, come ho detto, l'ira paleotipica è legata all'interfererenza di Alter col corpo di Ego, definisco invece l'indignazione come uno scarico di aggressività (usualmente repressa) causato dal mero bisogno di Tertius di scaricarla.

Questa mia proposta non è altro che una generalizzazione del concetto freudiano di Narzißismus der kleinen Differenzen:

\footnotetext{
${ }^{17}$ L'impeto, per come lo definisco in contrapposto all'ira, è tipico, dunque, dei fenomeni puramente attributivi individuati, che io sappia, per la prima volta da Jacek Kurczewski (1976a e 1976b, cfr. anche Fittipaldi 2012a: § 4.5 e $§ 4.12$ ). Kurczewski fa l'esempio del soldato in guerra, ma non vedo perché questa scoperta non possa venire generalizzata per tutti i fenomeni agonistici.
} 
Chiaramente non è facile per gli uomini rinunciare al soddisfacimento di questa loro tendenza a essere aggressivi; senza di essa non si sentirebbero tranquilli. Il vantaggio di un ambito piuttosto circoscritto di civiltà, il quale consente alla pulsione di sfogarsi animosamente contro coloro che ne sono al di fuori, non è affatto disprezzabile. È sempre possibile riunire un numero anche rilevante di uomini che si amino l'un l'altro fin tanto che ne restino altri per le manifestazioni di aggressività. Mi sono interessato una volta del fenomeno per cui comunità limitrofe e affini tra loro anche per altri versi si osteggiano e si scherniscono a vicenda, come gli Spagnoli e i Portoghesi, i Tedeschi del sud e del nord, gli Inglesi e gli Scozzesi, e molti altri. Denominai questo fenomeno "narcisismo delle piccole differenze", il che non contribuì molto alla sua spiegazione. Sappiamo ora che si tratta di un comodo, relativamente innocuo soddisfacimento dell'inclinazione aggressiva, in virtù del quale è facilitato l'accordo fra i membri di una comunità. [Freud 1929*, Cap. V: 601]

Freud distingue qui - come è del resto solito fare - le cause di questo fenomeno dalla sua funzione. La sua funzione, a suo avviso, è contribuire all'integrazione del gruppo, mentre la sua causa è il fatto che non sia facile per gli essere umani rinunciare alla soddisfazione della pulsione aggressiva (Befriedigung der Aggressionsneigung).

A mio avviso questo fenomeno può svolgere anche ulteriori funzioni o se si vuole usare un termine più neutrale - può produrre ulteriori effetti.

Una di queste funzioni è contribuire all'emersione di modelli di comportamento comuni a tutti i membri del gruppo. Rieccoci al concetto di modello usato da Ferrari e altri sociologi ${ }^{18}$.

Mi pare che si possa sintetizzare il modo in cui alcuni modelli emergono nel seguente modo:

- L'aggressività dei membri del gruppo è usualmente repressa.

- Reprimere questa aggressività richiede uno sforzo assai intenso da parte degli individui.

- Ciò implica un bisogno per la gran parte dei membri del gruppi (forse, mi verrebbe da aggiungere, soprattutto quelli di sesso maschile) di trovare un modo per scaricare questa aggressività.

- Un possibile modo, fra gli altri ${ }^{19}$, è rivolgerla all'indirizzo di comportamenti devianti.

È di fondamentale importanza sottolineare che non è assolutamente necessario che un comportamento sia stato già etichettato come deviante perché esso possa essere vissuto come idoneo a suscitare indignazione.

Invero, l'ipotesi qui avanzata è che a costituire la devianza di molti comportamenti è spesso proprio il bisogno di scaricare l'aggressività.

\footnotetext{
${ }^{18}$ Si veda per esempio Rehbinder (2000: $\left.§ 37\right)$, nella cui definizione di norma - peraltro fortemente indebitata con quella di Geiger - usa i termini Modell, Muster e Leitbild.

${ }^{19}$ Altri modi possono essere, ad esempio, le attività sportive già menzionate e la sublimazione. Sulla sublimazione dell'aggressività si veda, fra gli altri, Lorenz 1963* (cap. 14). Per quanto ne sappia Freud non usò mai questa espressione.
} 
Perché un comportamento diventi idoneo a suscitare indignazione può essere sufficiente la sua mera salienza cognitiva.

Questo approccio conduce a formulare 1'ipotesi sociologica per cui il conformismo si correla positivamente col livello di aggressività diffuso in una certa società ${ }^{20}$.

È evidente che qui abbiamo a che fare con qualcosa di più "di un modo relativamente innocuo di soddisfare la pulsione aggressiva".

Si pensi alla lapidazione. Questa pratica non richiede necessariamente un avente diritto "disonorato" (quale il padre o il marito di una donna), ma può avere luogo anche nel caso di condotte senza vittima. L'Antico Testamento, ad esempio, la prevedeva anche per il caso di violazione del divieto di astenersi dal lavoro il giorno del Sabato (Numeri 15,32-36) o per il caso di idolatria (Deutoronomio 17,2-5). Si tratta di casi in cui l'indignazione aveva anche l'effetto di contribuire all'integrazione del gruppo.

Si noti che l'indignazione svolge un ruolo fondamentale anche nel campo della cd. norma linguistica. Si pensi a come le persone colte possono reagire di fronte a un soggetto, magari con ambizioni culturali, che dica (o peggio ancora scriva) dasse, complementarietà o prendavamo.

Possiamo allora formalizzare la riduzione delle norme alla disposizione stabile a provare indignazione nel seguente modo:

$$
\begin{aligned}
& \text { (10) } x_{\text {ego }} \rightarrow \text { INDIGNAZIONE }_{\text {tertius }}\left(x_{\text {ego }}\right) \\
& \text { (11) } \neg x_{\text {ego }} \rightarrow \text { INDIGNAZIONE }_{\text {tertius }}\left(\neg x_{\text {ego }}\right)
\end{aligned}
$$

Nel caso (10) abbiamo un divieto mentre nel caso (11) abbiamo un obbligo. È appena il caso di sottolineare che neanche in questo caso è necessario che questi fenomeni emergano linguisticamente.

Stando ai presupposti qui adottati, per l'esistenza di una norma è quindi sufficiente che la disposizione a provare indignazione esista esclusivamente in Tertius. Il soggetto obbligato può essere completamente ignaro della "norma", e scoprirne l'esistenza solo dopo aver subito l'aggressività di Tertius, o di più Tertii. In altri termini, per l'esistenza di una norma è più che sufficiente l'esistenza di una siffatta disposizione in un Tertius.

Da un punto di vista sociologico, tuttavia, va sottolineato che l'ira e l'indignazione possono spesso combinarsi nel seguente triangolo:

$$
\text { (12) } x_{\text {ego }} \rightarrow\left[\operatorname{IRA}_{\text {alter }}\left(x_{\text {ego }}\right) \wedge \operatorname{INDIGNAZIONE}_{\text {tertius }}\left(x_{\text {ego }}\right)\right]
$$

Si pensi al caso previsto nell'Antico Testamento di lapidazione della donna che abbia mentito sulla propria verginità (Deuteronomio 22,13-20).

\footnotetext{
${ }^{20}$ Ricordo che Petrażycki formulò un'ipotesi parallela relativamente al formalismo giuridico allorché sostenne che si correlasse col grado di aggressività presente in una certa cultura.
} 
In questo caso, all'ira del marito e del padre si associa l'indignazione di tutta la comunità.

Sempre da un punto di vista strettamente sociologico va osservato che l'atteggiamento dei terzi è fondamentale anche nel caso in cui essi si astengano dallo scaricare direttamente la loro aggressività. Tale atteggiamento è infatti decisivo al fine di assicurare immunità all'autotutela del soggetto adirato (mutuo questa espressione da Sacco 2007: 299).

Un diverso fenomeno, che mi pare opportuno richiamare, al fine di evitare fraintendimenti, è il caso in cui Ego si indigni con Tertius perché quest'ultimo non riconosce il suo diritto (di Ego) nei confronti di Alter.

Karen Horney ha mostrato che anche in questo caso Alter, per Ego, può essere la realtà o il destino:

Take, for example, a businessman who is exasperated because a train does not leave at a time convenient for him. A friend, knowing that nothing is at stake, might indicate to him that he really is too demanding. Our businessman would respond with another fit of indignation $\left[{ }^{21}\right]$. The friend does not know what he is talking about. He is a busy man, and it is reasonable to expect a train to leave at a sensible time.

Surely his wish is reasonable. Who would not want a train to run at a schedule convenient to his arrangements? But - we are not entitled to it. This brings us to the essentials of the phenomenon: a wish or need, in itself quite understandable, turns into a claim. Its non-fulfillment, then, is felt as an unfair frustration, as an offense about which we have a right to feel indignant. [Horney 1950: 42, corsivi originali].

Un ulteriore fenomeno cui è opportuno accennare in questa sede è la $d e$ risione. Si tratta di un fenomeno strettamente connesso con lo scarico di aggressività; come segnalato dallo stesso Freud nel passo sopra riportato.

Com'è noto, Konrad Lorenz (1963*) sostenne che in origine il riso fosse una forma di scarico di aggressività. In questo caso si potrebbe considerare anche la derisione una forma di emozione etica capace di ipostatizzarsi in obblighi o divieti:

$$
\begin{aligned}
& \text { (13) } x_{\text {ego }} \rightarrow \text { DERISIONE }_{\text {ertius }}\left(x_{\text {ego }}\right) \\
& \text { (14) } \neg x_{\text {ego }} \rightarrow \text { DERISIONE }_{\text {ertius }}\left(\neg x_{\text {ego }}\right)
\end{aligned}
$$

Prima di passare alla discussione delle emozioni etiche masochistiche è opportuno concludere questa discussione delle emozioni etiche di tipo sadico con l'esame delle seguenti questioni:

1. Da un punto di vista ontogenetico, quando si sviluppano l'ira e l'indignazione?

${ }^{21}$ Come il lettore già sa, io userei il termine anger. 
2. La mia distinzione fra ira e indignazione è giustificata da un punto di vista etimologico?

3. Sono gli animali non umani capaci di provare ira e/o indignazione?

Per quanto riguarda la prima questione, va osservato che Anna Freud sostenne che $\mathrm{i}$ bambini sviluppano prima la capacità di provare quelle che qui chiamo emozioni etiche di tipo sadico rispetto al senso di colpa (A. Freud $1936^{*}: 128$ ss.).

Si potrebbe dire che ad avviso della studiosa i bambini sviluppano prima la capacità di vedere la pagliuzza nell'occhio altrui e solo in seguito - ammesso che davvero ciò accada (questo punto è di Anna Freud) - sviluppano la capacità di provare senso di colpa.

Per quanto riguarda la seconda questione (cioè se la mia scelta terminologica sia etimologicamente giustificata) va anzitutto precisato che - com'è noto - l'etimologia non ha nulla da insegnare in ordine al significato sincronico delle parole, giacché se le cose stessero in questo modo, nella lingua italiana, il vero significato di parole come casa o cavallo sarebbe "baracca" e "ronzino"...

Detto questo, la risposta alla questione (2) è negativa giacché indignatio in latino significava qualcosa di simile a ciò che qui ho chiamato ira domini. Infatti indignatio si riferiva alla reazione di ira di chi era stato trattato da indegno subendo una lesione dell'onore. Il termine indignitas in latino significava, sia l'“essere indegni", sia l'"essere stati trattati come degli esseri privi di dignità" (cfr. Thomas 2007: 165). Questo spiega perché il latino indignor avesse solo la forma medio-passiva (come l'italiano indignarsi, del resto). Il meccanismo sembra essere stato questo: vengo umiliato (indignor $_{1}$ ), quindi mi adiro (indignor ${ }_{2}$ ):

(15) $\operatorname{INDIGNAZIONE}_{\text {tertius }}\left(x_{\text {ego }}\right) \rightarrow \operatorname{IRA}_{\text {ego }}\left(\operatorname{INDIGNAZIONE}_{\text {tertius }}\left(x_{\text {ego }}\right)\right)$

È evidente che una discussione completa di questi fenomeni esigerebbe una discussione dei concetti di onore, dignità e vergogna. Questa discussione non può essere svolta in questa sede (ma sul concetto di vergogna vide infra). ${ }^{22}$

Veniamo ora alla terza questione, cioè se i nostri parenti animali siano capaci di provare ira e indignazione.

Non avendo competenze etologiche mi affido ad alcuni passi di de Waal nei quali egli discute alcune osservazioni svolte da lui e dai suoi collaboratori circa la correlazione fra la condivisione di cibo e le scariche di aggressività presso gli scimpanzé:

\footnotetext{
${ }^{22}$ La formula (15) invero è incompleta giacché vi è presumibilmente uno stadio intermedio consistente nella vergogna di Ego. Avremmo qui, dunque, una spirale vergogna-rabbia (shame-rage spiral) su cui vide infra.
} 
The rate of food distribution of each adult chimpanzee was defined as the number of food transfers to other adults divided by the possession time of a shareable quantity of food. This measure was compared with the number of aggressive acts received from other adults during food trials. [...] A negative correlation was found. [de Waal 1992: 45, see also de Waal 1997: 160]

In altri termini, nella situazione oggetto di osservazione, de Waal sostiene che quanto più spesso uno scimpanzé adulto risultava aver diviso con altri quantità di cibo sufficientemente grandi per essere condivise (tenendo conto della quantità di tempo che lo scimpanzé aveva avuto nella sua materiale disposizione), tanto più di rado avevano luogo scariche di aggressività. Di converso, quanto più di rado uno scimpanzé adulto risultava aver diviso quantità di cibo, tanto più spesso avevano luogo scariche di aggressività.

La cosa interessante è che:

when we looked further in this relation it became evident that the bulk of the aggression was received not by individuals in possession of food, but by those requesting food from others [..]. . [...] In particular it has been found that there is a] negative correlation between an individual's rate of food distribution and the probability of an aggressive response when this individual approached a food possessor. This result means that individuals that are least "generous" as food possessors were most likely to meet resistance on occasions when they had no food and tried to obtain a share from others. [De Waal 1992: 45]

Ora, se chiamiamo $c$ "chiedere cibo senza essere stati generosi in passato", possiamo formalizzare questo fenomeno nel modo seguente (dove $p$ sta per probabilità soprammedia):

(16) $c_{\text {ego }} \rightarrow p \cdot \operatorname{IRA} A_{\text {alter }}\left(c_{\text {ego }}\right)$

(17) $c_{\text {ego }} \rightarrow p \cdot \operatorname{INDIGNAZIONE}_{\text {tertius }}\left(c_{\text {ego }}\right)$

Stando alla terminologia qui adottata, mi pare che, qualora la reazione sia da parte di un soggetto cui Ego aveva in passato rifiutato del cibo, si possa parlare di ira di Alter. Per contro, nel caso in cui si tratti di un terzo si dovrà parlare di indignazione.

Si può addirittura azzardare una formulazione linguistica: è vietato agli individui non generosi chiedere cibo.

Un'ultima osservazione è qui necessaria. In casi come il (16) o il (17), nonché negli altri discussi supra, il fenomeno etico (o normativo) può essere esclusivamente in Alter o in Tertius. Se Ego si astiene dalla condotta $c$ solo perché teme la reazione aggressiva di Alter o di Tertius, Ego sta agendo esclusivamente per ansia sociale, e quindi l'agire di Ego non è un agire normativo nel nostro senso, ma tutt'al più un agire nomotropico nel senso di Conte (2011). È proprio per operare queste distinzioni che ritengo necessario respingere una definizione di norma quale quella proposta da Geiger. 
Il fenomeno etico è l'ira e l'indignazione, cioè lo scarico di un'aggressività usualmente repressa.

\section{Disposizioni etiche di tipo masochistico}

Fino ad ora abbiamo mostrato come le norme possano essere ridotte a disposizioni permanenti a provare ira e indignazione qualora si diano determinate circostanze.

L'ira e l'indignazione svolgono un ruolo fondamentale nell'ambito dei fenomeni giuridici definiti petrażyckianamente (supra n. 10). Anzi, si potrebbe dire che, petrażyckianamente, ubi ira, ibi ius. Per quanto riguarda l'indignazione, invece, abbiamo visto che essa può essere presente anche in ambito morale. In ambito agonistico (o puramente attributivo), invece, abbiamo fenomeni che ho chiamato impeto.

$\mathrm{Ne}$ segue che l'ira e l'indignazione sono i fenomeni di massimo interesse per il sociologo del diritto.

Nondimeno, sarebbe affrettato concludere che stabili disposizioni a provare vergogna e senso di colpa siano prive di interesse per il sociologo del diritto. Credo che sia ipotizzabile che la fisiologia del diritto - in contrapposto alla sua patologia - debba moltissimo a queste emozioni.

Dal momento che la distinzione fra vergogna e senso di colpa è stata oggetto di indagini enormi negli ultimi anni mi limiterò qui a esporre l'essenziale ai fini della ridefinizione di norma qui proposta.

L'aggettivo masochistico per riferirmi alla vergogna e al senso di colpa è una mia scelta. È ovvio il riferimento a Freud; fermo rimanendo che il concetto di masochismo morale ha per lui un significato molto più specifico (1924). Inoltre, come già detto, l'adozione di questa terminologia non impone l'adozione della teoria freudiana per cui il senso di colpa trarrebbe la propria "energia" dall'aggressività che il soggetto non può rivolgere verso l'esterno (1929, VII).

Vediamo ora (brevissimamente, visto che si tratta di cose largamente note) la differenza fra vergogna e senso di colpa.

La capacità di provare vergogna e senso di colpa emerge nell'interazione del bambino coi propri genitori (o le proprie figure di accudimento). I genitori sono persone che il piccolo, al contempo, ama e teme. La miscela di amore e timore è ciò che Bovet $\left(1925^{*}: 46\right)$ chiama rispetto; una definizione, questa, adottata da Jean Piaget (1932: 257 ss., 1932: 321 s.).

Il precursore sia del senso di colpa sia della vergogna è quella forma di ansia sociale che è la paura di essere puniti dai propri genitori o di perdere il loro amore. 
Qui finiscono le somiglianze fra vergogna e senso di colpa, giacché come risulterà evidente subito infra, nel caso della vergogna vi è la paura di essere indegni del loro amore, mentre nel caso del senso di colpa vi è la paura di aver perso il loro amore per aver fatto loro del male.

La gran parte della psicologia contemporanea distingue il senso di colpa dalla vergogna nel seguente modo: nel caso del senso di colpa il soggetto si focalizza su ciò che fece e, di regola, sul modo in cui ciò che fece incida o abbia inciso negativamente su altri individui. Nel caso della vergogna il soggetto prende in considerazione ciò che fece esclusivamente come sintomo della sua "vera" natura. Ne segue che nel caso del senso di colpa l'individuo desidererebbe rimediare a quel che fece, mentre nel caso della vergogna un simile desiderio non c'è.

Il paleotipo della colpa, come anticipato, è una condotta del bambino che il bambino capisce aver reso triste il proprio genitore. Il senso di colpa esige la capacità di provare empatia.

Per contro, il paleotipo della vergogna pare essere il rapporto del bambino con le proprie deiezioni ${ }^{23}$.

Sembra che il modo in cui i genitori "insegnano" al bambino a provare vergogna sia il disgusto (Michael Lewis 1992: 110). Utilizzando il formalismo qui introdotto potremmo sintetizzare questa modalità di socializzazione primaria nel seguente modo:

$$
\text { (18) } \operatorname{DISGUSTO}_{\text {genitore }}(\text { ego }) \rightarrow \text { VERGOGNA }_{\text {ego }}\left(\operatorname{DISGUSTO}_{\text {genitore }}(\text { ego })\right)
$$

È qui necessario dedicare qualche parola alla questione del perché gli animali umani provino disgusto nei confronti degli escrementi.

La migliore risposta è stata fornita da Rozin et al. (2008: 763), i quali anzitutto Rozin - hanno sintetizzato in modo originale l'enorme letteratura disponibile in argomento.

Sintetizzo al massimo. Il nucleo del disgusto è la bocca, bocca che è il custode dell'accesso al corpo. Da un punto di vista filogenetico, sembra che gli animali umani abbiano connesso il disgusto con la percezione di qualunque cosa ricordi loro il destino di morte e di decadimento fisico (si tenga presente che gli animali umani sembrano essere gli unici animali capaci di anticipare con largo anticipo il loro destino di morte e decadimento fisico). Il disgusto sarebbe stato quindi indirizzo, non solo nei confronti di possibili cause di morte, ma anche di possibili sintomi di morte (l'inconscio, come il pensiero magico, invero, - aggiunge chi scrive - non distingue molto bene fra cause e sintomi). Il disgusto, quindi, ha finito con l'investire tutto ciò che può ricordare agli esseri umani la loro natura animale. Il risultato sem-

\footnotetext{
${ }^{23}$ Sándor Ferenczy parlò, in generale, di moralità sfinterica (1925: 379).
} 
bra essere che, a seconda delle diverse culture, il disgusto può essere rivolto all'indirizzo degli esseri e delle condotto più disparate. Si pensi solo al ruolo fondamentale che il disgusto svolge nei sistemi castali.

Una questione su cui Rozin nei suoi diversi scritti mi pare non sottolineare (ma potrei sbagliare) è la stretta connessione fra il disgusto e la vergogna. Quando una figura di accudimento guarda il piccolo con un'espressione disgustata lo sta riducendo a un escremento e gli sta al contempo "insegnando" la capacità di provare vergogna.

Ecco perché nel caso della vergogna, a differenza del caso del senso di colpa, l'empatia sembra non svolgere alcun ruolo. La persona umiliata (cioè la persona che fa l'esperienza di vergogna) prova nei confronti di chi cagiona in lei tale esperienza tutto eccetto l'empatia. Anzi, come è stato largamente investigato a partire dalla pionieristica indagine di Helen Lewis (1971), le esperienze di vergogna possono innescare shame-rage spirals. Ciò perché la vergogna produce spesso in chi la prova quell'ira cui abbiamo già accennato quando abbiamo parlato dell'etimologia del termine latino indignor.

Fin qui ho discusso il disgusto per via della sua stretta connessione con la vergogna, tuttavia va rilevato che il disgusto dovrebbe venire discusso a parte come un'emozione etica di tipo sadico, distinta dall'indignazione e dall'ira. Certo, una connessione con l'ira potrebbe essere riscontrata nel fatto che la funzione originaria sia dell'ira sia del disgusto pare essere la protezione del corpo. Tuttavia, mentre il battito cardiaco, nel caso di ira, è accelerato, nel caso del disgusto è rallentato (Rozin et al. 2008: 763). Questa differenza mi pare da sola sufficiente ad accogliere l'opinione di Rozin che si tratti di fenomeni affatto diversi.

Queste notazioni su senso di colpa, vergogna e disgusto possono essere sufficienti ai nostri fini ${ }^{24}$.

Ora, come le norme possono essere ridotte a disposizioni permanenti a provare ira o indignazione, così le norme possono essere ridotte altresì, a seconda dei casi, a disposizioni permanenti a provare senso di colpa o vergogna, in determinate circostanze.

Quelle ipostatizzazioni, che chiamiamo obblighi o divieti, possono allora essere sintetizzate nei seguenti modi:

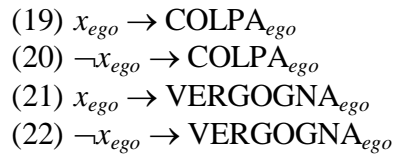

${ }^{24}$ Per una discussione più ampia della distinzione fra senso di colpa e vergogna si veda Fittipaldi 2012a. 
Dal momento che, come noto in letteratura psicologica, la vergogna, a differenza del senso di colpa, può essere anche contagiosa, occorre formalizzare anche questo fenomeno (mi limito ai divieti):

$$
\text { (23) } x_{\text {tertius }} \rightarrow \text { VERGOGNA }_{\text {ego }}\left(x_{\text {alter }}\right)
$$

In questo caso le norme vanno ridotte a disposizioni a provare vergogna o senso di colpa in determinate circostanze. Come nel caso dell'ira e dell'indignazione, l'esperienza del senso di colpa e della vergogna precede l'emersione di una disposizione stabile a provare senso di colpa e vergogna solo in determinate circostanze. Questa emersione produce a contrario l'emersione di un modello (cognitivo) di comportamento nel soggetto.

Fermo rimane che il modello esiste esclusivamente nella psiche del soggetto, e per di più, come detto, emerge solo a contrario; cioè dal desiderio del soggetto di evitare spiacevoli esperienze di vergogna e senso di colpa. Il modello emerge nella misura in cui il soggetto è in grado di identificare condotte più o meno simili che possono consentirgli di evitare simili esperienze.

Si potrebbe domandare se vi siano prototipi (in contrapposto ai paleotipi) di ciò che è in grado di suscitare vergogna o senso di colpa. Stando alla ricerca psicologica, sembrerebbe che qualunque tipo di situazione possa, a seconda del soggetto considerato, suscitare vergogna o senso di colpa (cfr. Tangney \& Dearing 2002: 17).

A me pare, tuttavia, che alcune condotte sia quasi inconcepibile che diano luogo a senso di colpa. Penso in particolare alle condotte con le quali si manifesta ignoranza. Penso anzitutto, ma non solo, alla violazione di cd. "norme" linguistiche.

Ne segue che se l'ira è esclusiva, per non dire costitutiva, dei fenomeni giuridici, la questione se la vergogna o il senso di colpa siano più o meno correlati coi fenomeni giuridici o con quelli morali (sempre distinti petrażyckianamente) è un poco più sfumata. Mi pare che il massimo che si può azzardare sia l'ipotesi per cui la vergogna sia più correlata coi fenomeni morali.

Da quanto detto sopra a proposito del disgusto segue altresì che certe "norme" vanno ridotte alla disposizione stabile a provare disgusto all'indirizzo di certe condotte:

$$
\text { (24) } x_{\text {ego }} \rightarrow \text { DISGUSTO }_{\text {tertius }}\left(x_{\text {ego }}\right)
$$

In casi di questo tipo, ovviamente, nel caso in cui Ego non provi vergogna, l'unico fenomeno etico si trova in Tertius.

È qui opportuno esaminare brevemente la questione se gli animali siano in grado si provare vergogna o senso di colpa. 
Per quanto riguarda la vergogna, vista la sua stretta connessione col disgusto, pare ipotizzabile che gli animali non umani siano incapaci di farne esperienza.

Per quanto riguarda il senso di colpa, invece, è stato sostenuto da Konrad Lorenz che essi sarebbero capaci di provarla. Leggiamo come de Waal commenta le idee di Lorenz:

Konrad Lorenz [...] related how one of his dogs, Bully, accidentally bit [his] hand when he tried to break up one of fiercest dog fights he had ever seen. Even though Lorenz did not reprimand him and immediately tried to reassure and pet him, Bully was so upset by what he had done that he suffered a complete nervous breakdown. For days he was virtually paralyzed, and uninterested in food. He would lie on the rug breathing shallowly, an occasional deep sigh coming from his tormented soul. Anyone who had not seen the incident would have thought he had come down with a deadly disease. For weeks Bully remained extremely subdued.

Lorenz noted that his dog had never bitten a person before, so could not have relied on previous experience to decide that he had done something wrong. Perhaps he had violated a natural taboo on inflicting damage to a superior, which normally (among members of his species) could have the worst imaginable consequences. If so, instead of saying that Bully felt guilty, it might be more appropriate that he expected punishment, perhaps even expulsion from the pack. [de Waal 1996: 106, corsivo mio]

Mi pare di poter sommessamente osservare che l'esperienza di Bully, per quanto diversa dal senso di colpa tipico degli animali umani, mi pare differire da esso solo in parte.

Abbiamo infatti visto che il senso di colpa origina da una situazione nella quale:

1. il piccolo teme una punizione da parte della figura di accadimento;

2. il piccolo teme di perdere l'amore della figura di accadimento;

3. il piccolo prova empatia per un male inferto alla figura di accudimento.

Se questo è il paleotipo della colpa, mi pare che nel caso di Bully ci troviamo in una situazione che lo ricorda da vicino. Quanto questo paleotipo disti, per Bully, da una vera e propria interiorizzazione, chi scrive non ha le competenze per dire.

\section{Disposizioni etiche di tipo narcisistico}

Con l'espressione emozioni etiche di tipo narcisistico mi riferisco a emozioni etiche il cui paleotipo è costituito dal tentativo da parte del piccolo di divenire come la propria figura di accudimento. Dal momento che il piccolo concepisce le proprie figure di accudimento come alcuni adulti concepiranno la divinità, cioè come dotate di ogni perfezione (Bovet 
$1925^{*}$ ), fare ciò che la figura di accudimento fa equivale per il piccolo a essere partecipe delle sue perfezioni.

Già Joseph Sandler osservò (1960) che quando il piccolo decide di lavarsi le mani, non solo conserva l'amore della mamma facendo ciò che lei desidera, ma altresì diventa pulito come sua madre. Questo è ciò che Sandler chiama narcissistic advantage.

Sia l'orgoglio sia l'ammirazione vanno considerati emozioni etiche di tipo narcisistico.

Il termine orgoglio di rado si sente riferito a norme. Ciò perché in letteratura psicoanalitica l'orgoglio viene più frequentemente riferito all'ioideale (ciò che il soggetto deve diventare) piuttosto che al super-io (ciò che il soggetto deve, può o non deve fare). Tuttavia, se l'io-ideale costituisce ciò che l'individuo dovrebbe essere, più che fare (questo è il caso del superio), si capisce che siamo in piena normatività; per lo meno nel senso qui proposto.

In genere, negli adulti sani, l'orgoglio viene provato nel caso in cui si riesca a svolgere un compito difficile. Orgoglio può essere provato da un soldato che obbedisca a ordini cui ben pochi obbedirebbero. Orgoglio può essere provato da un debitore che paghi un debito prescritto. Orgoglio può essere provato da un amico che aiuti un amico in difficoltà.

In tutti questi casi, l'approccio qui adottato implica che gli unici fenomeni reali siano l'emozione in sé e - ammesso che vi sia - la stabile disposizione a provarla. Non si può parlare di norme a meno che non si accetti di usare il termine norma nel senso qui proposto di disposizione stabile a provare emozioni superegoiche (o etiche, o normative) ${ }^{25}$.

In questi casi possiamo allora ridurre le norme di obbligo o le norme proibitive nei seguenti modo:

$$
\begin{aligned}
& \text { (25) } x_{e g o} \rightarrow \text { ORGOGLIO }_{e g o} \\
& \text { (26) } \neg x_{\text {ego }} \rightarrow \text { ORGOGLIO }_{\text {ego }}
\end{aligned}
$$

In questi casi, a differenza del caso del senso di colpa e della vergogna, l'emozione etica svolge il ruolo, non di una sanzione negativa, bensì di una sanzione positiva per il soggetto. Tornando alla questione di che cosa sia e come emerga il modello di cui parla Ferrari, in questi casi dobbiamo concludere che questo modello emerge direttamente, non a contrario.

\footnotetext{
${ }^{25} \mathrm{Si}$ potrebbe domandare se io non commetta una petitio principii nel parlare di debito come ho fatto poco supra nel testo. Una risposta dettagliata a questa domanda richiederebbe moltissimo spazio. Basti dire che l'approccio qui sostenuto porta ad affermare che se nessun partecipante ha la stabile disposizione a provare le emozioni qui descritte non si può parlare di debito. Ciò che può esistere o essere esistito è la storica integrazione di fattispecie la cui integrazione è generalmente idonea a far venire ad esistenza le disposizioni di cui parlo nel testo.
} 
Per quanto riguarda il caso di orgoglio per astensione/astinenza si pensi alle privazioni per ragioni religiose nelle quali il soggetto, attraverso simili attività, mira a conseguire un'identificazione narcisistica col proprio Dio (Mancia 2010).

L'ammirazione può essere definita come una forma di identificazione narcisistica con un individuo che porta a termine qualcosa di difficile.

L'esperienza di quest'emozione è di solito incompatibile con l'esperienza dell'invidia, ma non si può scartare l'ipotesi che per lo meno in certi casi l'ammirazione sia una formazione reattiva nei confronti di esperienze di invidia che il soggetto non può ammettere a se stesso.

Si potrebbe sintetizzare quanto detto affermando che l'ammirazione $\grave{e}$ l'orgoglio dei terzi.

Possiamo formalizzare questo tipo di norme nel seguente modo:

(27) $\mathrm{x}_{\text {ego }} \rightarrow$ AMMIRAZIONE tertius

(28) $\frown_{\frown} \mathrm{X}_{\text {ego }} \rightarrow$ AMMIRAZIONE ${ }_{\text {tertius }}$

Queste formule significano che in Tertius esiste una norma secondo la quale Ego dovrebbe (27) o non dovrebbe (28) compiere $x$. Ovvio che in casi del genere la norma può esistere anche esclusivamente nella psiche di Tertius.

$\mathrm{Va}$ osservato che anche un avente diritto (uso una terminologia ipostatizzante) può talvolta provare ammirazione o gratitudine nei confronti di un soggetto che tenga una condotta a suo favore che la gran parte dei soggetti non terrebbe.

Ora, al fine di evitare fraintendimenti, va sottolineato che se l'ammirazione è un sentimento superegoico, la gratitudine non lo è assolutamente.

La gratitudine non è altro che l'ansia che chi ci ha fatto del bene possa cambiare idea. Quest'ansia è perfettamente compatibile con l'irreversibilità del tempo, dal momento che l'irreversibilità del tempo pertiene al principio di realtà e la psiche profonda non sa nulla di tale irreversibilità. Questo è ciò che chiamo gratitudine in senso stretto. Fermo rimane che nulla esclude che da un atto di generosità possa nascere anche sincero affetto; ma questo fenomeno ha ben poco a che fare con la gratitudine.

Infine va rilevato che la gratitudine può essere oggetto di emozioni etiche nel senso che è perfettamente possibile che Ego si senta in colpa o si vergogni per il fatto di non provare gratitudine nei confronti di Alter, o che Alter si adiri che il fatto che Ego non provi gratitudine nei suoi confronti, o che Tertius di indigni per il fatto Ego non provi gratitudine nei confronti di Alter. 


\section{Conclusioni}

Da quanto precede segue che ciò che spesso si chiama norma può essere una o più di una delle seguenti disposizioni (mi limito agli obblighi):

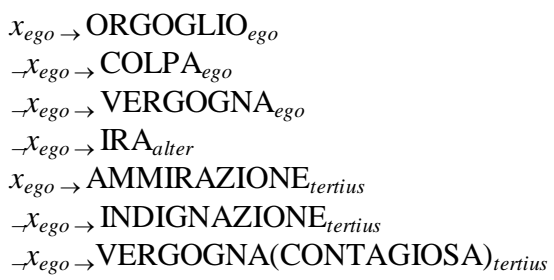

Da un punto di vista sociologico può accadere che i diversi partecipanti abbiano disposizioni etiche compatibili, come sintetizzato dalla tab. 1.

Ma la situazione rappresentata nella tab. 1 è solo una eventualità. ${ }^{26}$ L'approccio qui adottato implica che i fenomeni etici, e soprattutto quelli giuridici, possano essere incompatibili, e quindi polemogeni. Ciò per via del ruolo che le emozioni etiche sadiche possono svolgere. Si tratta della tesi di Petrażycki (1909-10), che chi scrive si limita qui ad articolare alla luce delle ricerche svolte posteriormente al suo insegnamento.

Una concezione della norma quale quella qui proposta impone al sociologo del diritto di indagare:

1. in quale misura determinate regolarità comportamentali siano causate da disposizioni etiche, e,

2. nel caso di regolarità comportamentali causate da disposizioni etiche, quali siano le disposizioni etiche prevalenti.

Tab. 1. Eventuale compatibilità di disposizioni etiche

\begin{tabular}{|c|c|c|}
\hline Ego (obbligato) & Alter (avente diritto) \\
\hline $\begin{array}{c}\text { colpa in caso di } \neg x \\
\text { vergogna in caso di } \neg x \\
\text { orgoglio in caso di } x\end{array}$ & ira in caso di $\neg x$ \\
\hline Ego deve fare $x$ & \\
\hline & Tertius (spettatore) \\
\hline $\begin{array}{c}\text { indignazione in caso di } \neg x \\
\text { vergogna contagiosa in caso di } \neg x \\
\text { ammirazione in caso di } x\end{array}$ \\
\hline
\end{tabular}

${ }^{26} \mathrm{Si}$ noti che eventuale è anche la presenza di Alter (cioè di avente diritto). 
Questo porta a includere la nota distinzione fra shame cultures e guilt cultures, ma altresì a non escludere che possano esservi società nelle quali la gran parte delle regolarità comportamentali siano dovute a emozioni etiche, non di tipo masochistico, bensì di tipo sadico, quali l'ira e l'indignazione. Da italiano, mi domando se le regolarità comportamentali nel nostro Paese non vadano spiegate - quando non spiegabili in termini economici - come dovute a stabili disposizioni a provare emozioni etiche sadiche (diverse dall' indignazione).

Ricordo (cfr. supra) che queste distinzioni possono essere decisive per spiegare fenomeni come il formalismo giuridico. È questa un'ipotesi, come già detto, riconducibile all'insegnamento di Petrażycki.

Rispetto alla distinzione fra shame cultures e guilt cultures desidero puntualizzare che l'approccio qui sostenuto, ovviamente, esclude radicalmente che questa distinzione sia sovrapponibile con quella fra società a sanzione esterna e società a sanzione interna. Si tratta di una tesi che risale a Margaret Mead:

In societies in which the individual is controlled by fear of being shamed, he is safe if no one knows of his misdeeds [...] but the individual who feels guilt must repent and atone for his sin. [Mead 1937: 494]

È una tesi che, nonostante il fondamentale lavoro di Piers e Singer (1953) è tuttora dato ascoltare presso antropologi del diritto. Che la vergogna possa essere completamente privata è tesi ovvia dal punto di vista strettamente psicologico (p.es. Lewis 1995: 75 ss.). Oltretutto si pensi che esistono addirittura disturbi della personalità, quale il disturbo narcisistico, che emergono come formazioni reattive nei confronti di questo tipo di esperienza privatissima!

Le società a sanzione esterna di cui parla Mead andrebbero piuttosto chiamate, stando alle proposte qui presentate, anger societies o indignation societies (né le anger societies coincidono necessariamente con le indignation societies).

Un ultima considerazione.

La mia proposta (lo ripeto, fortissimamente debitrice nei confronti dell'insegnamento di Petrażycki) porta a ripensare completamente il rapporto fra diritto e forza (in un modo paradossalmente convergente rispetto a quello di Kelsen).

Il diritto non si serve della forza. Piuttosto, è la disposizione a scaricare un'aggressività altrimenti repressa nei casi di ira iuris e ira domini a costituire il nucleo psicologico di quell'ipostatizzazione chiamata diritto soggettivo.

Si badi bene, il termine diritto soggettivo va inteso in modo estremamente ampio, tale da coprire l'ira di un poliziotto dinanzi a un automobilista 
che non faccia quanto il poliziotto gli comandi (cfr. Petrażycki 1909-10: 216, 1909-10*: 136). ${ }^{27}$

Una questione complessa con la quale mi pare opportuno concludere è se sia possibile una società in cui le regolarità comportamentali non siano causate da nessun tipo di emozione etica, neppure dalla paura dell'ira e dell'indignazione altrui (che in questi caso, lo ricordo, sono le uniche emozioni etiche). È una domanda difficilissima la risposta alla quale è collegata, a mio avviso, con la legittimazione della sociologia del diritto come scienza autonoma.

\section{Riferimenti bibliografici}

Bovet, Pierre, $1925^{*}$. Le sentiment religieux et la psychologie de l'enfant. English translation The Child's Religion. London \& Toronto: Dent \& Sons 1928.

Ceretti, Adolfo, \& Lorenzo Natali. Cosmologie violente. Percorsi di vite criminali. Milano: Cortina.

Conte, Amedeo G., 2011. Sociologia filosofica del diritto. Torino: Giappichelli.

de Waal, Frans B. M., 1992. Aggression as a Well-integrated Part of Primate Social Relationships: A Critique of Seville Statement on Violence. In James Silverberg \& J. Patrick Gray (eds.), Aggression and Peacefulness in Humans and Other Primates. New York - Oxford: Oxford University Press 1992.

-, 1997. Good Natured. The Origins of Right and Wrong in Humans and Other Animals. Cambridge (MA) - London: Harvard University Press.

Durkheim, Émile, 1897*. Le suicide. English translation Suicide. New York: The Free press of Glencoe.

Ferenczi, Sándor, 1925. Psychoanalysis of Sexual Habits. The International Journal of Psycho-Analysis, 6: 372 ss.

Ferrari, Vincenzo, 2005. Diritto e società. Roma-Bari: Laterza.

Fittipaldi, Edoardo 2012a. Everyday Legal Ontology. A Psychological and Linguistic Investigation within the Framework of Leon Petrazycki's Theory of Law. Milan: Led.

—, 2012b. Psicologia giuridica e realismo. Leon Petrażycki. Milan: Led.

Freud, Anna, 1936*. Das Ich und die Abwehrmechanismen. English Translation The Ego and the Mechanisms of Defence. New York: International Universities Press. Inc. 1946.

Freud -, 1916. Einige Charaktertypen aus der psychoanalytischen Arbeit. Pubblico dominio.

—, 1924. Das ökonomische Problem des Masochismus. Pubblico dominio.

—, 1929. Das Unbehagen in der Kultur. Pubblico dominio.

${ }^{27}$ Petrażycki parla di negodovanie (sdegno) poiché non andò oltre la distinzione fra appulsione e repulsione etiche. 
- $1929^{*}$. Das Unbehagen in der Kultur. Traduzione italiana Il disagio della civiltà in Sigmund Freud (a cura di Cesare Musatti), Opere. Torino: Bollati Boringhieri, 1989.

Friedman, Lawrence, 1975. The Legal System. A Social Science Perspective. New York: Russell Sage.

Geiger, Theodor, $1964^{2}$. Vorstudien zu einer Soziologie des Rechts. Neuwied am Rhein \& Berlin: Luchterhand.

Horney, Karen, 1950. Neurosis and Human Growth. The Struggle toward SelfRealization. New York \& London: Norton \& Company.

Kurczewski, Jacek, 1976a. Ambiguous Reciprocity. The Polish Sociological Bulletin, 2: 5 ss.

—, 1976b. Due dimensioni del diritto: diritto positivo o intuitivo e diritto ufficiale e non ufficiale. Sociologia del diritto, (1), 1: 29 ss.

Lewis, Helen B., 1971. Shame and Guilt in Neurosis. New York: International University Press.

Lewis, Michael. 1992. Shame. The Exposed Self. New York: The Free Press.

Lorenz, Konrad, 1963*. Das sogenannte Böse. Zur Naturgeschichte der Aggression. English translation On Aggression. New York \& London: Harvest.

Luhmann, Niklas, $1969^{*}$. Normen in soziologischer Perspektive. Traduzione italiana parziale Aspettative e norme, in Giuseppe Lorini \& Lorenzo Passerini Glazel Filosofie della norma. Torino: Giappichelli: 2012.

Mancia, Maurizio, 2010. Il narcisismo. Il presente deformato allo specchio. Torino: Bollati Boringhieri.

Mead, Margaret (ed.), 1937. Cooperation and Competition among Primitive Peoples. Boston: Beacon Press, 1961.

Petrażycki, Leon, 1909-10. Teorija prava i gosudarstva v svjazi s teoriej nravstvennosti. $2^{\text {nd }}$ ed, Sankt Peterburg: Ekateringofskoe Pečatnoe Delo.

-, 1909-10*. Teorija prava i gosudarstva v svjazi s teoriej nravstvennosti. $2^{\text {nd }}$ ed. Partial English translation in Petrażycki 1955†.

—, 1955 †* (Nicholas S. Timasheff ed.). Law and Morality. Cambridge (Ma): Harvard University Press.

Piaget, Jean, 1932. Le jugement moral chez l'enfant. Parigi: Presses Universitaires de France.

—, 1932*. Le jugement moral chez l'enfant. Traduzione inglese The Moral Judgement of the Child. Londra: Routledge \& Kegan Paul.

Rehbinder, Manfred, 2000. Rechtssoziologie. Monaco di Baviera: Beck.

Rozin, Paul, Jonathan Haidt \& Clark R. McCauley, 2008. Disgust. In M. Lewis, J.M. Haviland-Jones \& L.F. Barrett (a cura di), Handbook of Emotions, New York: Guildford Press, $2008^{3}$.

Sacco, Rodolfo, 1991. Legal Formants: A Dynamic Approach to Comparative Law. The American Journal of Comparative Law, 39, 1, 343 ss.

—, 1992. Introduzione al diritto comparato. Torino: UTET.

-, 1995. Mute Law. The American Journal of Comparative Law, 43, 3: 455 ss.

—, 1997. Perché l'armato ubbidisce all'inerme? Saggio sulla legittimazione del potere. Rivista di diritto civile, 1997, I, 1 ss. 
—, 2007. Antropologia giuridica. Bologna: il Mulino.

Sandler, Joseph, 1960. On the Concept of Superego. The Psychoanalytic Study of the Child, 15: 128 ss.

Schlicht, Ekkehart, 1998. On Custom in the Economy. Oxford: Clarendon Press.

Shaver, Philip, Judith Schwartz, Donald Kirson \& Cary O’Connor, 1987. Emotion Knowledge: Further Exploration of a Prototype Approach. Journal of Personality and Social Psychology, (52) 6: 1061 ss. DOI: 10.1037//00223514.52.6.1061

Silverberg, James \& J. Patrick Gray (eds.), 1992. Aggression and Peacefulness in Humans and Other Primates. New York \& Oxford: Oxford University Press.

Smith, Adam, $1790^{6}$. The Theory of Moral Sentiments. Pubblico dominio.

Tangney, June P., \& Ronda L. Dearing, 2002. Shame and Guilt. London: The Guildford Press.

Thomas, Jean-François, 2007. Déshonneur et honte en latin: Etude Semantique. Leuven : Peeters. 